\title{
Queloide bilateral secundario a otoplastia tratado mediante escisión y colgajos queloideos
}

\author{
Bilateral keloids secondary to otoplasty treated by excision \\ and keloids flaps
}

\section{A. Pérez-Espadero, F. J. Escudero Nafs}

\section{RESUMEN}

Los queloides son cicatrices de aspecto tumoral que pueden aparecer hasta varios años después de la herida original, y se extienden más allá de los confines de ésta. La oreja prominente es la deformidad auricular estética más frecuente. La otoplastia para su corrección logra resultados altamente satisfactorios. Sin embargo, no está exenta de complicaciones, entre las que hay que considerar el desarrollo de queloides. Existen muchas técnicas para la extirpación y la posterior cobertura. La técnica del colgajo queloideo consiste en la escisión del queloide preservando una capa delgada de su cubierta cutánea, unida por un pedículo a la piel normal, para reparar el defecto. El colgajo queloideo es una solución sencilla y efectiva para la cobertura de defectos importantes tras la extirpación completa de grandes queloides. Se presenta un caso clínico ilustrativo, secundario a otoplastia, del empleo de dicho procedimiento.

Palabras clave. Queloide. Oreja. Otoplastia. Reconstrucción de oreja. Colgajo queloideo.

\begin{abstract}
Keloids are tumour-like scars that appear some years after the original wound, and which spread beyond the limits of the scar. Prominent ears are the most common deformity of the earlobe. The correction of this abnormality with otoplasty achieves very satisfactory results. On the other hand, otoplasty could entail several adverse effects, of which the keloid scar must be considered. There are numerous techniques for the removal of keloids and for performing a subsequent coverage. The keloid flap consists in the excision of the keloid scar preserving a thin layer of skin which covers the keloid, with a pedicle attached to the normal skin, in order to close the defect. The keloid flap is an effective and simple solution for the coverage of large defects after complete giant keloid eradication.
\end{abstract}

Key words. Keloid. Ear. Otoplasty. Ear reconstruction. Keloid flap.
Servicio de Cirugía Plástica y Reparadora. Complejo Hospitalario de Navarra. Pamplona.

Recepción: 23 de octubre de 2012

Aceptación provisional: 4 de febrero de 2013

Aceptación definitiva: 18 de marzo de 2013

\section{Correspondencia:}

A. Pérez-Espadero

Servicio de Cirugía Plástica y Reparadora

Complejo Hospitalario de Navarra

C/ Irunlarrea, 4

31008 Pamplona

E-mail: apespadero@hotmail.com 


\section{INTRODUCCIÓN}

Los queloides son cicatrices de aspecto tumoral que pueden aparecer hasta varios años después de la herida original, se extienden más allá de los confines de ésta y tienden a recidivar tras la escisión quirúrgica $^{1,2,3}$. En la oreja se pueden desarrollar a partir de diversos tipos de heridas, como piercing, incisiones quirúrgicas, laceraciones y quemaduras ${ }^{4}$. La oreja prominente, llamada también oreja en asa o en soplillo, es la deformidad auricular estética más frecuente y a menudo se asocia a problemas psicológicos durante la infancia. La otoplastia para su corrección logra resultados altamente satisfactorios en la mayoría de los $\operatorname{casos}^{5}$. Sin embargo, no está exenta de complicaciones, entre las cuales hay que considerar el desarrollo de cicatrización hipertrófica e incluso de queloides a partir de la herida incisional ${ }^{6,7}$. Los queloides de gran tamaño que invaden la oreja producen deformidades con repercusión estética importante. Los defectos secundarios a su extirpación se han tratado con diversidad de técnicas reconstructivas, algunas de ellas complejas. La técnica del colgajo queloideo consiste en la escisión del queloide preservando una capa delgada de su cubierta cutánea, unida por un pedículo a la piel normal, para reparar el defecto ${ }^{8}$. Esta técnica se ha descrito para la reconstrucción de defectos de oreja, cara, tronco y genitales secundarios a la extirpación de queloides ${ }^{8}$. Se presenta un caso clínico ilustrativo, secundario a otoplastia, del empleo de dicho procedimiento.
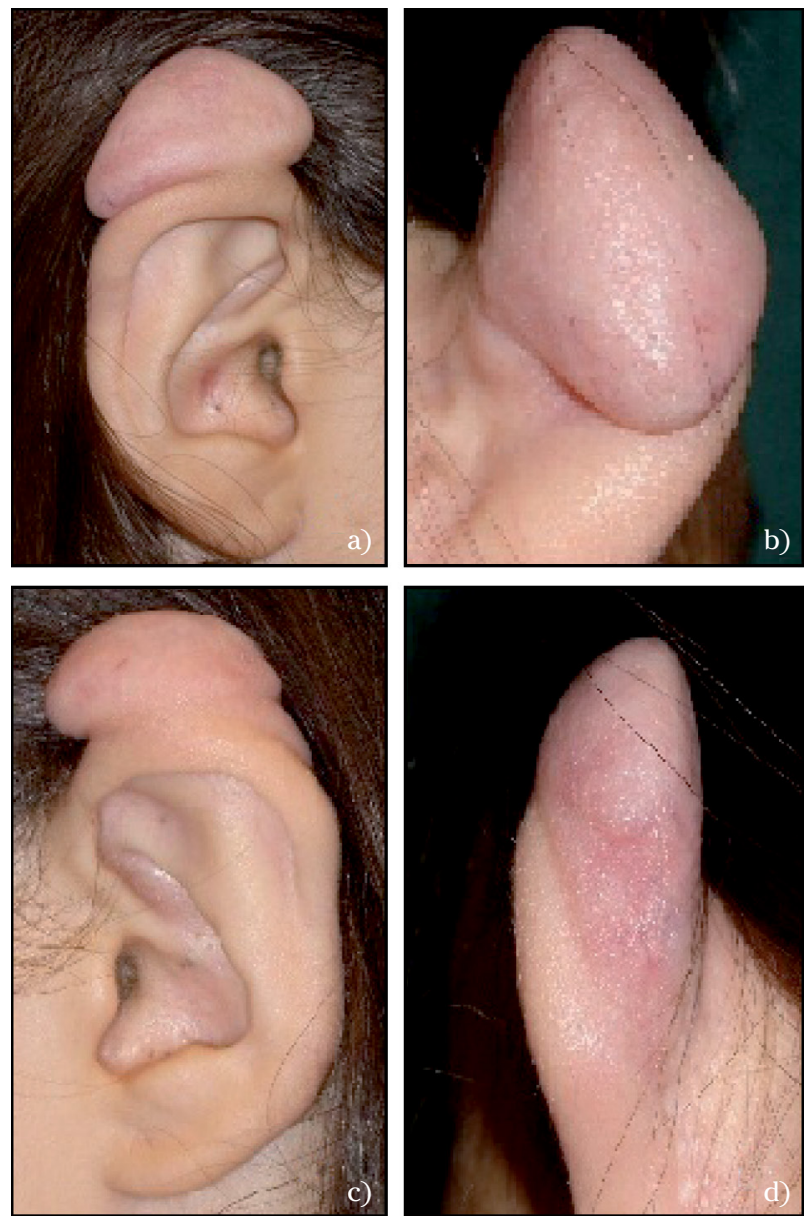

Figura 1. Queloide de la oreja derecha: Aspecto lateral (a). Aspecto posteromedial (b). Queloide de la oreja izquierda: Aspecto lateral (c). Aspecto posteromedial (d). 


\section{CASO CLÍNICO}

Niña de 12 años de edad, con antecedente de orejas prominentes tratadas mediante otoplastia a los 8 años. En ambas orejas se desarrolló progresivamente un queloide a partir del año de la intervención. Los queloides invadían el borde superior y cara posterior de los polos superiores. El derecho tenía un tamaño horizontal de 3,2 $\mathrm{cm}$ y vertical de $2 \mathrm{~cm}$, y el izquierdo de $2,7 \mathrm{~cm}$ x $1,5 \mathrm{~cm}$ respectivamente (Fig. 1). La paciente no refería dolor ni prurito. Se indicó extirpación dado el gran tamaño de las lesiones, con repercusión estética y psicológica. La intervención se realizó con anestesia general. El tratamiento quirúrgico consistió en la técnica siguiente: se realizó una incisión en la cara posterosuperior del queloide, a partir de la cual de disecó un colgajo delgado de la cubierta cutánea queloidea, man- teniendo un pedículo de unión a la piel normal en la cara anterior de la oreja. Tras levantar el colgajo de un tamaño adecuado se realizó extirpación del resto del queloide. El colgajo fue suturado al defecto sin tensión (Fig. 2). El tamaño de los colgajos fue de $2,5 \mathrm{~cm} \times 1,5 \mathrm{~cm}$ en la oreja derecha, y de $2 \mathrm{~cm}$ x $1 \mathrm{~cm}$ en la oreja izquierda. En el estudio anatomopatológico se comprobó que los nódulos queloideos estaban recubiertos por epidermis, observándose atrofia de anejos cutáneos. La cirugía se asoció a otros tratamientos, empleados habitualmente para la prevención de recidivas: inyección intraoperatoria de corticosteroide (acetónido de triamcinolona) en el lecho quirúrgico, aplicación de gel de silicona en las cicatrices resultantes y presoterapia con una banda elástica. El gel de silicona y la presoterapia se emplearon durante un año tras la intervención. La paciente fue controlada periódicamente.
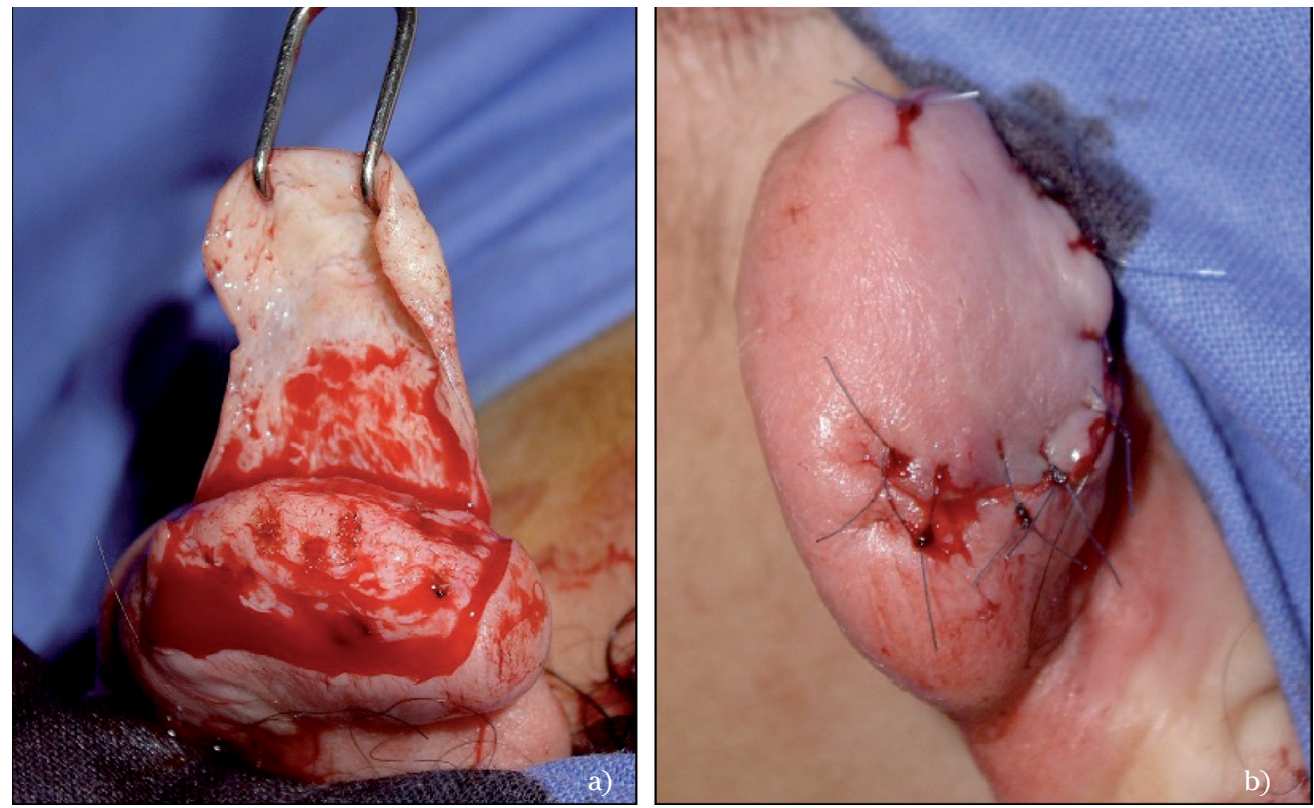

Figura 2. Disección de colgajo queloideo (a). Reparación del defecto tras extirpación del resto del queloide (b).

El colgajo de la oreja derecha presentó una dehiscencia pequeña de sutura, la cual curó por segunda intención a las pocas semanas mediante tratamiento tópico. No se desarrollaron necrosis u otras complicaciones. Se logró una reparación de los defectos satisfactoria, con restitución del contorno normal de las orejas. En la oreja dere- cha se desarrolló una recidiva a los 5 años de la intervención, formándose un queloide en la zona operada de $1,5 \mathrm{~cm} \times 1 \mathrm{~cm}$, el cual fue tratado con el mismo procedimiento quirúrgico. En la oreja izquierda no se ha producido recidiva. A los 18 años de edad, ambas orejas presentan buen aspecto y la paciente realiza controles periódicos (Fig. 3) 

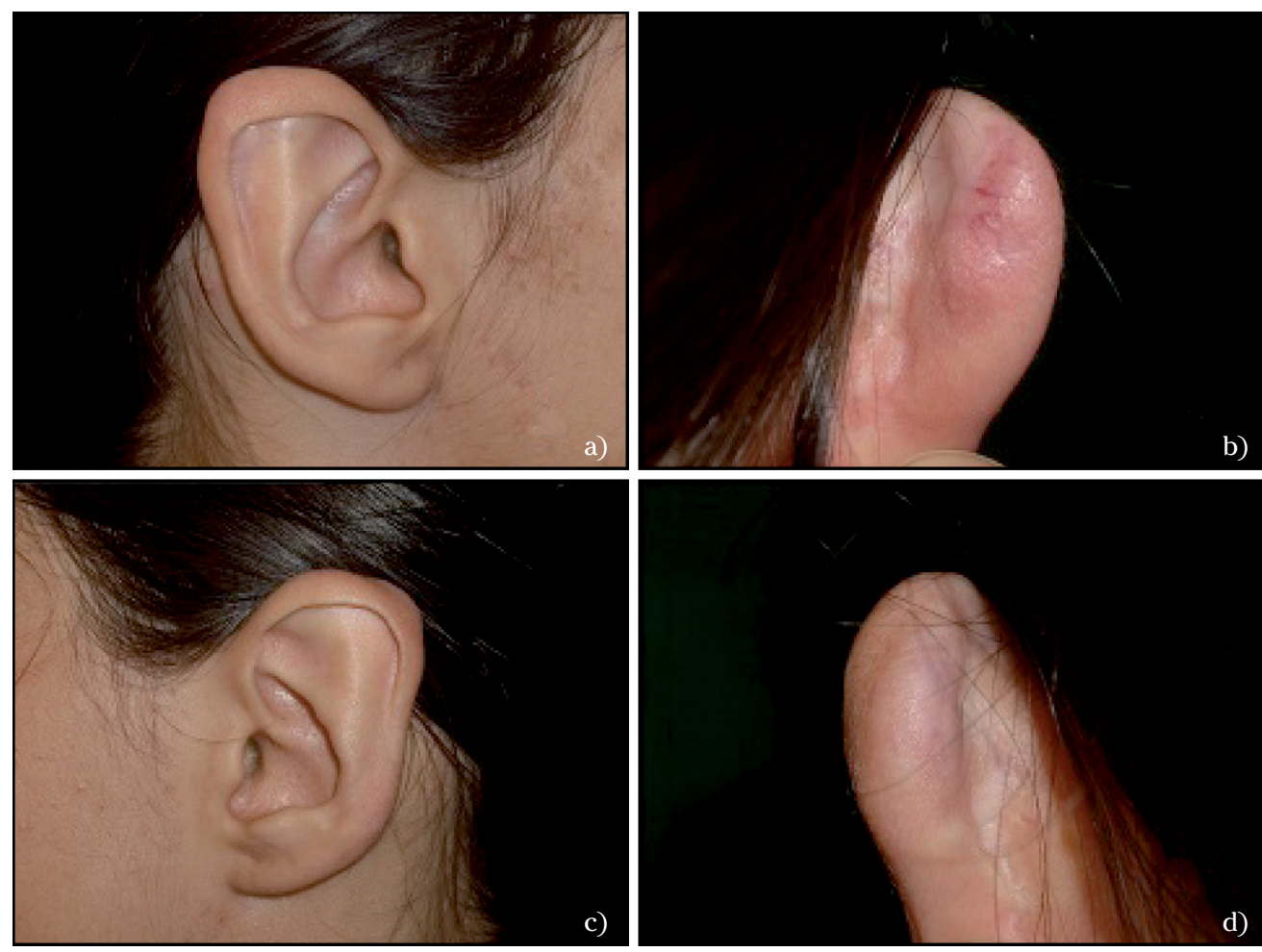

Figura 3. Resultado a los 6 años de la intervención. Oreja derecha: Aspecto lateral (a). Aspecto posteromedial (b). Oreja izquierda: Aspecto lateral (c). Aspecto posteromedial (d).

\section{DISCUSIÓN}

La corrección de orejas prominentes proporciona un alto grado de satisfacción ${ }^{5}$. Aunque infrecuente, hay que considerar el riesgo de cicatriz hipertrófica o de queloi$\mathrm{de}^{6,7}$. Calder y Naasan, en una revisión sobre la morbilidad de la otoplastia en 562 casos, con edades comprendidas entre 3 y 67 años, comprobaron una incidencia de 12 casos $(2,1 \%)^{6}$. Los queloides tienen más tendencia a desarrollarse durante etapas de mayor actividad fisiológica hormonal, tales como la pubertad. En la serie de otoplastia de Calder y Naasan6, 11 de los 12 pacientes que presentaron queloides como complicación, tenían 14 o menos años de edad.

Se han considerado diversos factores etiopatogénicos de la cicatrización hiper- trófica o queloidea, entre los que destacan los siguientes: 1) desequilibrio entre la síntesis (aumentada) y la degradación (disminuida) del colágeno, por aumento de la actividad de factores de crecimiento, tales como TGF- $\beta$ y PDGF, que estimularían la síntesis e inhibirían la degradación de la matriz extracelular, y/o por disminución de la apoptosis de fibroblastos; 2) tensión mecánica en la sutura de heridas, que estimularía la proliferación de fibroblastos y la síntesis de colágeno; 3) reacción inmunológica anormal a la lesión dérmica o a la materia sebácea, habiéndose considerado que la cicatrización patológica se podría comportar como una enfermedad de tejido conectivo autoinmune $\mathrm{e}^{1,2,3}$. Con respecto a la otoplastia, se considera que una escisión cutánea postauricular excesiva, con sutura bajo tensión, puede favorecer el desarrollo 
de una cicatrización patológica. Se puede prevenir este factor mediante una escisión cutánea conservadora o bien evitándola?

Existen muchas opciones de tratamiento de los queloides, a menudo empleadas combinadamente y con diversos grados de efectividad, lo que sugiere que no existe una modalidad terapéutica única e ideal. La incidencia de recidiva sobrepasa el $80 \%$ si el tratamiento se limita exclusivamente a la cirugía ${ }^{4}$, por lo que es importante la elección de un tratamiento combinado eficaz. Es frecuente que la extirpación se combine con otros tratamientos, como inyección intralesional de corticosteroides, aplicación tópica de silicona, presoterapia y radioterapia, entre otros, con los que se ha demostrado una reducción de la incidencia de recidiva ${ }^{1-4,9}$.

Los defectos secundarios a la escisión de queloides en las orejas se han tratado con diversas opciones, como cicatrización por segunda intención, sutura directa, injertos y colgajos cutáneos ${ }^{10}$. En el caso de defectos de gran tamaño es conveniente evitar una cicatrización por segunda intención o una sutura cutánea a tensión, ya que ambas favorecen el desarrollo de cicatrices patológicas. Los injertos o colgajos cutáneos obtenidos de otras zonas de la oreja o distantes conllevan nuevas cicatrices con su morbilidad asociada.

La técnica del colgajo queloideo consiste en la escisión del queloide preservando una capa delgada de su cubierta cutánea, unida a la piel normal a través de un pedículo $^{8,10-13}$. Con el colgajo obtenido se repara el defecto sin tensión en la sutura. En los estudios que han descrito su aplicación se considera que el colgajo está constituido por piel (epidermis y dermis superficial) distendida por el crecimiento del queloide, y nutrida por un plexo vascular tenue $\mathrm{e}^{8,10,11,13}$.

En series clínicas sobre el empleo de este colgajo, asociado ${ }^{13}$ o no $^{8,10}$ a tratamientos complementarios, se ha observado una incidencia de recidiva variable, oscilando entre $12,5^{8}$ y $60 \%{ }^{13}$. En nuestro caso recidivó parcialmente el queloide en la oreja cuyo colgajo presentó dehiscencia y curación por segunda intención, lo cual podría haber favorecido el desarrollo de una cica- trización excesiva, aunque ésta no se manifestó hasta pasados varios años del tratamiento. La utilización de parte del queloide con finalidad reconstructiva plantea la incertidumbre de si la piel queloidea puede ser origen de una recidiva. Luo y $\operatorname{col}^{14}$ han demostrado, en muestras de queloides obtenidas de pacientes, que los fibroblastos de la región central del queloide proliferan más rápido in vitro que los periféricos, mientras que éstos, correspondientes a las regiones superficial y basal, no difieren de fibroblastos normales respecto al crecimiento celular. En los colgajos queloideos se preserva la zona más superficial, cuyos fibroblastos tendrían un equilibrio más normal entre proliferación y muerte celular (apoptosis), con menos probabilidad de recidiva en la zona reparada y en la línea de sutura.

Como conclusión de nuestra experiencia con el caso descrito y de la revisión bibliográfica, consideramos que la técnica del colgajo queloideo en reconstrucción auricular aporta una solución sencilla y efectiva para la cobertura de defectos importantes tras la extirpación completa de grandes queloides, sin necesidad de recurrir a injertos o colgajos obtenidos de otras zonas, cuya morbilidad puede ser considerable.

\section{BIBLIOGRAFÍA}

1. RAHBAN SR, GARNER WL. Fibroproliferative scars. Clin Plast Surg 2003; 30: 77-89.

2. Al-Attar A, Mess S, Thomassen JM, Kauffman CL, DAVISON SP. Keloid pathogenesis and treatment. Plast Reconstr Surg 2006; 117: 286-300.

3. Gauglitz GG, Korting HC, Pavicic T, Ruzicka T, JESCHKE MG. Hypertrophic scarring and keloids: Pathomechanisms and current and emerging treatment strategies. Mol Med 2011; 17 : 113-125.

4. Park TH, Seo SW, Kim JK, Chang CH. Outcomes of surgical excision with pressure therapy using magnets and identification of risk factors for recurrent keloids. Plast Reconstr Surg 2011; 128: 431-439.

5. EsCUDERo FJ. Corrección de las orejas prominentes mediante la otoplastia de Chongchet. Ventajas e inconvenientes. Revista de Divul- 
gación Científica de la AECEP (Asociación Española de Cirugía Estética Plástica) 2007; 2: 42-47.

6. CALDER JC, NaAsan A. Morbidity of otoplasty: a review of 562 consecutive cases. Br J Plast Surg 1994; 47: 170-174.

7. Owsley TG, BiggerstafF TG. Otoplasty complications. Oral Maxillofacial Surg Clin N Am 2009, 21: 105-118.

8. LeE Y, Minn KW, BaEK RM, Hong JJ. A new surgical treatment of keloid: keloid core excision. Ann Plast Surg 2001; 46: 135-140.

9. Gutiérrez C, López FJ, Lara J, Cervantes JP, MárQuez E, Morales D. Protocolo de tratamiento de cicatrices queloides en el pabellón auricular del Hospital General Dr. Manuel Gea González. Cir Plast Iberolatinoam 2012; 38: 49-54.

10. KIm DY, KIm ES, Eo SR, KIm KS, LeE SY, Сно BH. A surgical approach for earlobe keloid: ke- loid fillet flap. Plast Reconstr Surg 2004; 113 : 1668-1674.

11. Napoleone C, Giovannelli R, Capoccetti E, Zara $\mathrm{L}$, FRANCHI R. Use of keloid cutis as reconstructive flap of the auricle after keloid excision. Plast Reconstr Surg 2003; 111: 518519.

12. Suliman MT. The keloid fillet flap. Plast Reconstr Surg 2005; 116: 337-338.

13. Valerón P, Dehesa L, Vilar J, Domínguez J, Gómez J, Carretero G. Tratamiento quirúrgico del queloide recidivante de pabellón auricular mediante "colgajo en filete". Actas Dermosifiliogr 2010; 101: 235-241.

14. Luo S, Benathan M, Raffoul W, Panizzon RG, EgLoff DV. Abnormal balance between proliferation and apoptotic cell death in fibroblasts derived from keloid lesions. Plast Reconstr Surg 2001; 107: 87-96. 